\title{
Streptomyces clavuligerus sp. nov., a $\beta$-Lactam Antibiotic Producer
}

\author{
C. E. HIGGENS and R. E. KASTNER
}

The Lilly Research Laboratories, Eli Lilly and Company, Indianapolis, Indiana 46206

\begin{abstract}
A new species of Streptomyces which produces two new cephalosporin antibiotics is named and described. Sporophores are produced on an extensive aerial mycelium and consist of networks of short, sympodially branched hyphae. One to four spores are borne usually on short, club-shaped side branches. Sporophores eventually segment to form chains of spores. The spores are oblong to slightly cylindrical, smooth-walled, and gray to grayish green en masse. The species name proposed, Streptomyces clavuligerus, refers to the production of club-like side branches.
\end{abstract}

A previously undescribed streptomycete isolated from a South American soil sample by a selective isolation procedure was found to produce two new cephalosporin antibiotics, 7-(5-amino-5-carboxyvaleramido)-3-carbamoyloxymethyl-3-cephem-4-carboxylic acid and 7-(5-amino-5-carboxyvaleramido)-3-carbamoyloxy methy 1-7-methoxy-3-cephem-4-carboxylic acid (6). Another organism, Streptomyces lipmanii (Waksman and Curtis) Waksman and Henrici, strain NRRL 3584, was isolated from a different soil sample from the same locality and likewise was found to produce a new cephalosporin antibiotic, 7-(5-amino-5-carboxyvaleramido)-7-methoxycephalosporanic acid (6). Both organisms also produce penicillin $\mathrm{N}$, an antibiotic previously reported from a streptomycete by Miller et al. (Bacteriol. Proc., p. 32, 1962). The former organism is believed to be a new species in the genus Streptomyces Waksman and Henrici, and in this report the new species is named, described, and assigned a type strain.

\section{MATERIALS AND METHODS}

Isolation of bacterial strain. The organism was selected from a series of soil isolations that were plated at dilutions of $1: 10^{3}$ to $1: 10^{5}$ on a modified Pridham and Gottlieb basal medium (7), supplemented with $0.5 \%$ glucose. Unsterilized nystatin (E. R. Squibb and Sons, New York, N.Y.) at $30 \mathrm{mg} / \mathrm{liter}$ was added to the melted agar before dilution plates were poured. Inoculated plates were incubated at $30 \mathrm{C}$ for 10 days.

Method. The methods and media recommended by the International Streptomyces Project (ISP; 10) were used primarily, along with several supplementary tests. Stock slant cultures were maintained on ISP no. 2 (yeast-malt) agar. These slants, as well as subsequent cultures used in this study, were incubated at $30 \mathrm{C}$. Inocula were prepared by transferring a loopful of spores from an agar slant into a 250 -ml flask containing $70 \mathrm{ml}$ of tryptone-yeast extract broth (7). Flasks were incubated on a rotary shaker [2-inch $(5 \mathrm{~cm})$ stroke, $250 \mathrm{rev} / \mathrm{min}$ ] for $72 \mathrm{hr}$. Broth cultures were macerated for $30 \mathrm{sec}$ in an homogenizer, centrifuged, and then suspended in $0.85 \%$ sterile saline. Inocula for carbon-utilization tests were washed twice and suspended in saline.

Microscopic observations were made on cultures that were grown from 4 to 21 days on ISP no. 4 (inorganic salts-starch), Bennett's, and ISP no. 5 (glycerol-asparagine) media. Sporophore morphology was observed on undisturbed plate cultures, and slide mounts were stained with $0.1 \%$ Acid Fuchsin in lactic acid-glycerol (1:1). A single-stage, carbon replicaplatinum shadowing technique (3) was used to obtain electron micrographs.

Colony characteristics were observed from petri dish cultures on 11 media after 7 and 14 days of incubation (Table 1). The formulas for nutrient, Bennett's, glucose-asparagine, Czapek's, calcium malate, and tyrosine agars are those of Waksman (15). Tomato paste-oatmeal agar was prepared as suggested by Pridham et al. (8). Temperature requirements were determined on ISP no. 2 (yeast-malt) agar. Gelatin liquefaction was observed at 14 days on a medium composed of $12 \%$ gelatin, $0.5 \%$ beef extract (Difco), and $0.5 \%$ peptone (Difco). The medium was refrigerated after incubation to detect liquefaction. The method of Boone and Pine (2) was employed to prepare cells for amino acid analysis. Determinations were made with a Beckman model 120 amino acid analyzer. The procedure of Becker et al. (1) was used for chromatographic detection of the isomers of diaminopimelic acid. 


\section{RESULTS AND DISCUSSION}

A total of 1,852 strains, composed mainly of streptomycetes, was selected from 235 South American soil samples. Three strains were found to produce $\beta$-lactam antibiotics. One was a strain of Streptomyces lipmanii (Waksman and Curtis) Waksman and Henrici, which was deposited in the Agricultural Research Service Collection, Peoria, Ill., as NRRL 3584. Two strains isolated from another soil sample produced antibiotics found to be similar on the basis of paper chromatographic data; however, one strain did not grow on transfer, and a complete characterization could not be made.
The remaining streptomycete was also deposited in the Agricultural Research Service Collection as NRRL 3585 and in the American Type Culture Collection, Rockville, Md., as ATCC 27064.

Although only one strain of this organism has been isolated thus far, it is quite distinct (see below) from the previously described species of Streptomyces and is therefore here regarded as belonging to a new species, for which we propose the name Streptomyces clavuligerus. A description of this organism follows.

Streptomyces clavuligerus sp. n., cla.vu.li'-
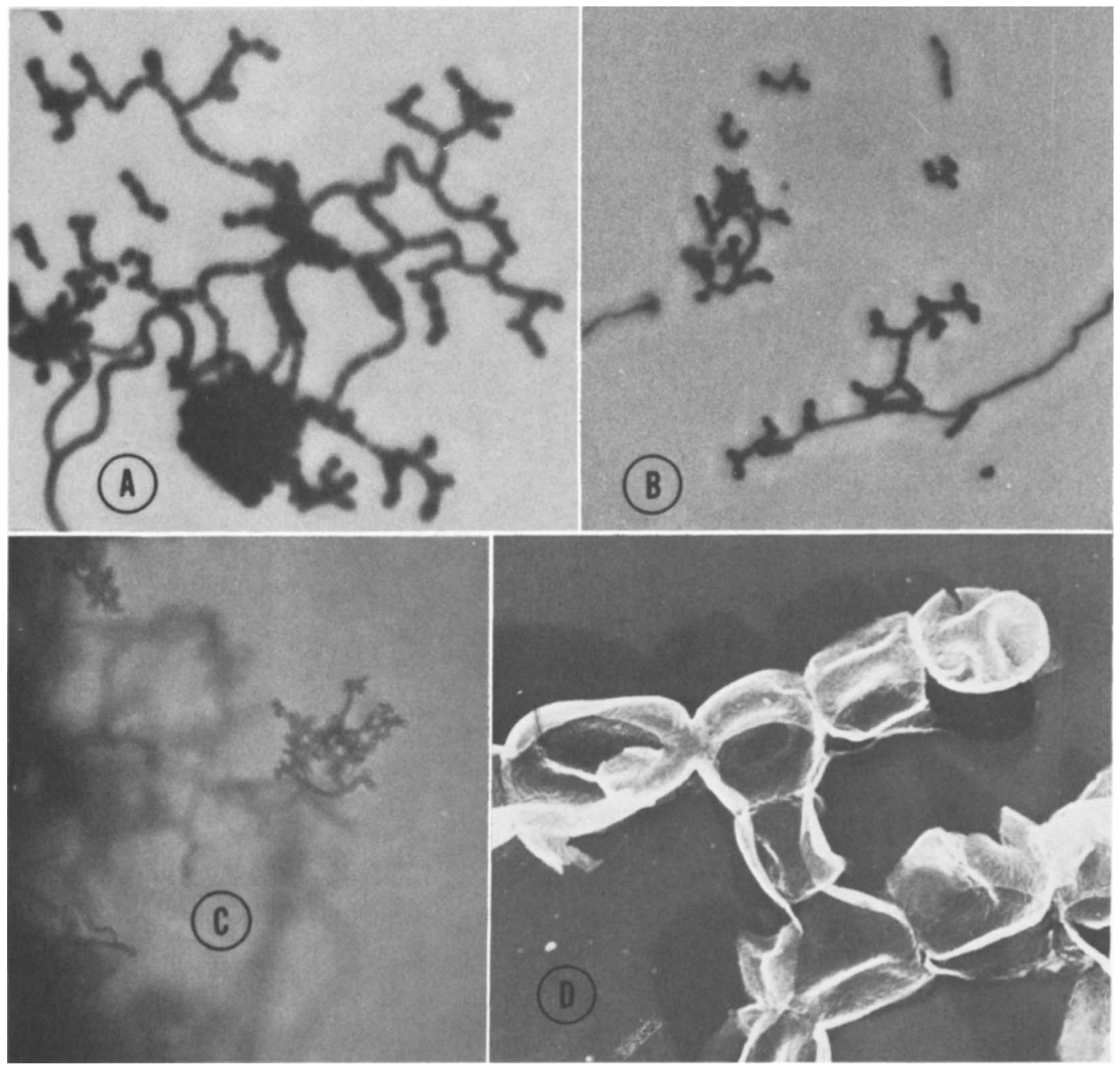

Fig. 1. Morphology of Streptomyces clavuligerus NRRL 3585. A and B, Sporophore morphology; stained impression slide mount ( $A$ is $\times 1,700$ and $B$ is $\times 770$ ); $C$, sporophore habitat: undisturbed agar plate, $\times 380 ; D$, electron micrograph of spores, $\times 20,000$. 
ger.us. L. fem. n. clavula little club; L. suffix -igerus bearing; M. L. adj. clavuligerus bearing little clubs.

Produces an aerial mycelium which is composed of a network of sympodially branched, aerial hyphae that eventually segment into spores. Short, clavate, side branches are formed that usually produce from one to four spores each (Fig. 1A, 1B, and 1C). The spore chain morphology is classified in the Rectus-flexibilis section of Pridham et al. (9).

Spores are oblong to short-cylindrical,

TABLE 1. Cultural characteristics of Streptomyces clavuligerus

\begin{tabular}{l} 
Medium \\
\hline $\begin{array}{l}\text { ISP no. } 2 \\
\text { (yeast-malt extract agar) }\end{array}$ \\
ISP no. 3 \\
(oatmeal) \\
ISP no. 4 \\
(inorganic salts-starch) \\
ISP no. 5 \\
(glycerol-asparagine) \\
Tomato paste-oatmeal
\end{tabular}

Bennett's agar

Czapek's agar

Glucose-asparagine

Tyrosine agar

Nutrient agar

Calcium malate
Characteristics $^{a}$

Growth abundant, reverse grayish yellow [12K3] ; aerial mycelium abundant, light grayish olive (GN) $1 \frac{1}{2}$ ge [21B 1] ; no soluble pigment

Growth moderate, reverse pale yellow [11C1] ; aerial mycelium fair, white (W) $b$ [27A 1$]$; no soluble pigment

Growth abundant, reverse grayish yellow [12B2] ; aerial mycelium moderate, medium gray (GY) 2fe [45A 1]; no soluble pigment

Growth fair, reverse pale yellow green [10B 1]; aerial mycelium fair, white (W) $a$; no soluble pigment

Growth abundant, reverse grayish yellow [11E4]; aerial mycelium moderate, light grayish olive (GN) 1/2ig [21B1]; no soluble pigment

Growth abundant, reverse light yellow [11J2]; aerial mycelium abundant, dark grayish green (GN) 24\%/2ih [23A3]; no soluble pigment

Scant growth

Growth moderate, reverse pale yellow green [10B1] ; aerial mycelium fair, white (W) $b$ [27A1] ; no soluble pigment

Growth moderate, reverse pale yellow [10B2] ; aerial mycelium moderate, yellowish gray (GY) $2 d c$ [10A2]; no soluble pigment

Growth fair, reverse pale yellow green [10B 1] ; aerial my celium sparse, white; no soluble pigment

Growth abundant, reverse pale yellow green [10B 1] ; aerial mycelium fair, white (W) $a$

\footnotetext{
${ }^{a}$ Color names were assigned according to the methods of the Intersociety Color Council (4). Letters in parentheses refer to the Tresner and Backus color series (14); color-tab designations are italicized. Figures in brackets refer to colors from the color blocks of Maerz and Paul (5).
} 
averaging 0.64 by $1.53 \mu \mathrm{m}$ in size, with smooth spore surfaces when observed by electron microscopy (Fig. 1D). Neither fragmentation of hyphae nor formation of spores occurred in the substrate mycelium.

Cultural characteristics are presented in Table 1. Aerial mycelium is dark grayish green on media with abundant sporulation. The color ranges from white to gray, to light grayish white on other media. Substrate mycelia vary from pale yellow to grayish yellow. No soluble pigment is produced on any of the 11 media used. The culture is assigned to the Gray (GY) and Green (GN) series of Tresner and Backus (14). The Maerz and Paul (5) color block most similar to the spore color en masse is 21-B1, and the light grayish olive color of the ISCCNBS (4) designation method corresponds to this color block.

The culture grows over a $p \mathbf{H}$ range of 5.0 to 8.5. Growth does not occur at either $p \mathrm{H} 4.0$ or 9.0. Sporulation occurs from $p \mathrm{H} 5.0$ to 6.5 and is most abundant at $p \mathrm{H} \mathrm{6.0.}$

Whole-cell hydrolysates contain L-diaminopimelic acid, glycine, aspartic acid, alanine, glutamic acid, and leucine as major constituents.

Physiological tests are recorded in Table 2, and carbon-utilization patterns are recorded in Table 3.

A comparison of minimum inhibitory concentrations of several cultures to $\beta$-lactam antibiotics is given in Table 4 . $S$. clavuligerus does not appear to be significantly more resistant to
TABLE 3. Utilization of various carbon compounds by Streptomyces clavuligerus ${ }^{a}$

\begin{tabular}{lc}
\hline \multicolumn{1}{c}{ Compound } & Utilization \\
\hline L-Arabinose & - \\
Rhamnose & - \\
D-Fructose & - \\
D-Xylose & - \\
Melezitose & $(-)$ \\
Raffinose & - \\
D-Glucose & - \\
Cellobiose & - \\
Maltose & + \\
Sucrose & - \\
Cellulose & - \\
I-Inositol & $(+)$ \\
D-Mannitol & - \\
\hline
\end{tabular}

a Symbols: +, positive utilization; (+), probable utilization; (-), questionable utilization; - , no utilization.

$\beta$-lactam antibiotics than several unrelated streptomycetes.

A comparison of this organism was made with the published descriptions of 300 type strains of Streptomyces species $(11,12,13)$. Of 56 organisms chosen for morphological similarity, $S$. catenulae Davisson and Finlay and $S$. ramulosis Ettlinger et al. were the most similar on the basis of sporophore morphology. However, $S$. catenulae differs from $S$. clavuligerus by

TABLE 2. Physiological properties of Streptomyces clavuligerus

\begin{tabular}{|c|c|}
\hline Property observed & Characteristics \\
\hline Action on milk & No coagulation; clearing in 17 days \\
\hline Nitrate reduction & Negative \\
\hline Gelatin liquefaction & None \\
\hline Growth response to $p \mathrm{H}$ changes & $\begin{array}{l}p \mathrm{H} 5.0-6.0 \text { in optimum range for growth; } \\
\text { growth but no sporulation from } p \mathrm{H} \\
7.0-8.5\end{array}$ \\
\hline $\begin{array}{l}\text { Melanin production } \\
\text { (Peptone-iron agar and tryp- } \\
\text { tone-yeast extract broth) }\end{array}$ & None \\
\hline Temperature requirements & $\begin{array}{l}\text { Growth and sporulation good from } 26 \text { to } 30 \mathrm{C} \text {; } \\
\text { no growth at } 37 \mathrm{C} \text { or above }\end{array}$ \\
\hline $\begin{array}{l}\text { Major constituents of whole-cell } \\
\text { hydrolysates }\end{array}$ & $\begin{array}{l}\text { L-Diaminopimelic acid, glycine, glutamic } \\
\text { acid, aspartic acid, alanine, and leucine }\end{array}$ \\
\hline
\end{tabular}




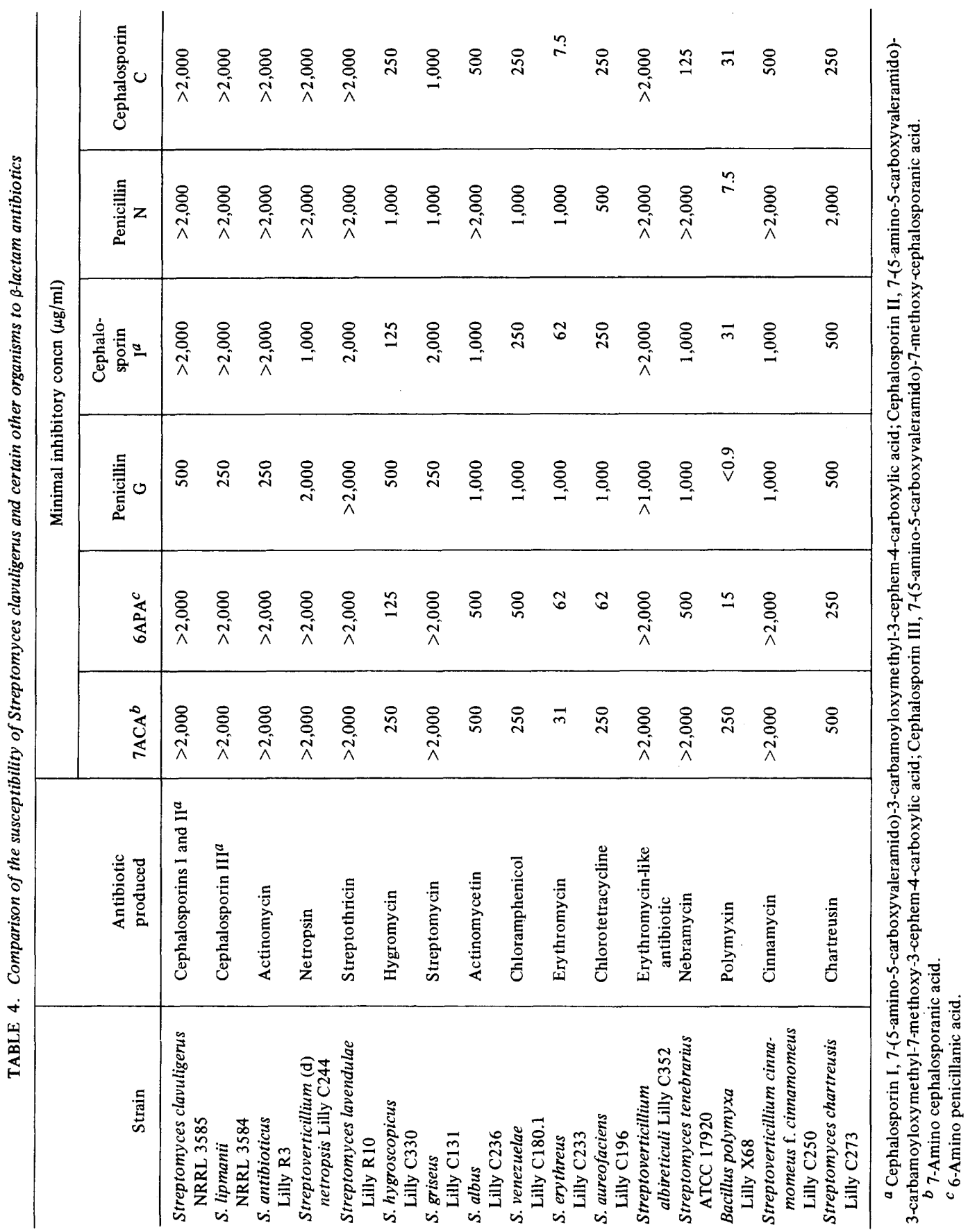


producing a different vegetative color, tight spirals, and a soluble pigment on some media. It also differs considerably in its ability to utilize certain carbon sources and in the nature of the antibiotics it produces. $S$. ramulosis produces a reddish brown to dark brown vegetative color, a red to violet pigment on some media, and also differs in the utilization of several carbon sources.

Habitat: Soil.

The type strain is NRRL 3585 (= ATCC 27064). (Because the species description given above is based on a single strain, NRRL 3585, it also serves as the description of the type strain.)

\section{ACKNOWLEDGMENTS}

We are grateful to $R$. A. Schlegel for preparing the electron micrograph and to R. Ellis for providing the amino acid analyses.

\section{LITERATURE CITED}

1. Becker, B., M. P. Lechevalier, R. E. Gordon, and H. A. Lechevalier. 1964. Rapid differentiation between Nocardia and Streptomyces by paper chromatography of whole-cell hydrolysates. Appl. Microbiol. 12:421-423.

2. Boone, C. J., and L. Pine. 1968. Rapid method for characterization of actinomycetes by cell wall composition. Appl. Microbiol. 16:279-284.

3. Bradley, D. E. 1960. Replica techniques in applied microscopy. J. Roy. Microsc. Soc. 79:101-118.

4. Kelly, D. L., and D. B. Judd. 1955. The ISCCNBS method of designating colors and a dictionary of color names. U.S. Dept. of Commerce Circ. 553. Washington, D.C.

5. Maerz, A., and M. R. Paul. 1950. Dictionary of color. McGraw-Hill Book Co., Inc., New York.
6. Nagarajan, R., L. D. Boeck, M. Gorman, R. L. Hamill, C. E. Higgens, M. M. Hoehn, W. M. Stark, and J. G. Whitney. 1971. $\beta$-Lactam antibiotics from Streptomyces. J. Amer. Chem. Soc. 93:2308-2310.

7. Pridham, T. G., and D. Gottlieb. 1948. The utilization of carbon compounds by some Actinomycetales as an aid for species determination. J. Bacteriol. 56:107-114.

8. Pridham, T. G., P. Anderson, C. Foley, L. A. Lindenfelser, C. W. Hesseltine, and R. G. Benedict. 1957. A selection of media for maintenance and taxonomic study of Streptomyces. Antibiot. Ann. 1956-57, p. 947-953.

9. Pridham, T. G., C. W. Hesseltine, and R. G. Benedict. 1958. A guide for the classification of streptomycetes according to selected groups. Appl. Microbiol. 6:52-79.

10. Shirling, E. B., and D. Gottlieb. 1966. Methods for characterization of Streptomyces species. Int. J. Syst. Bacteriol. 16:313-340.

11. Shirling, E. B., and D. Gottlieb. 1968. Cooperative description of type cultures of Streptomyces. II. Species descriptions from first study. Int. J. Syst. Bacteriol. 18:69-189.

12. Shirling, E. B., and D. Gottlieb. 1968. Cooperative description of type cultures of Streptomyces. III. Additional species descriptions from first and second studies. Int. J. Syst. Bacteriol. 18:279-392.

13. Shirling, E. B., and D. Gottlieb. 1969. Cooperative description of type cultures of Streptomyces. IV. Species descriptions from the second, third and fourth studies. Int. J. Syst. Bacteriol. 19:391-512.

14. Tresner, H. P., and E. J. Backus. 1963. System of color wheels for streptomycete taxonomy. Appl. Microbiol. 11:335-338.

15. Waksman, S. A. 1961. The actinomycetes, vol. 2 . Classification, identification and descriptions of genera and species. The Williams \& Wilkins Co., Baltimore. 